\title{
Is monotherapy with a beta-lactam antibiotic inferior to combination therapy with a beta-lactam antibiotic and rifampicin in treating prosthetic joint infections? A retrospective study.
}

\section{Bamo Jalal (D BamoSamalJalal@gmail.com )}

Sjaellands Universitetshospital Koge https://orcid.org/0000-0002-0893-4698

Veronika Murlasits

Sjaellands Universitetshospital Koge

Jonas B. Boel

Herlev Sygehus: Herlev Hospital

Jesper Sonntag

Hillerød Sygehus: Nordsjaellands Hospital

Morten Michelsen

Gentofte University Hospital: Gentofte Hospital

Hans Gottlieb

Herlev Sygehus: Herlev Hospital

Magnus Arpi

Herlev Sygehus: Herlev Hospital

Lene Nielsen

Herlev Sygehus: Herlev Hospital

Research Article

Keywords:

Posted Date: February 2nd, 2022

DOI: https://doi.org/10.21203/rs.3.rs-1273767/v1

License: (c) (i) This work is licensed under a Creative Commons Attribution 4.0 International License. Read Full License 


\section{Abstract}

Background: The use of rifampicin in Prosthetic joint infections (PJI's) is widely used, although the evidence of its effectiveness is sparce. The aim of this study was to retrospectively examine if monotherapy with a beta-lactam antibiotic was inferior to combination therapy with a beta-lactam antibiotic and rifampicin in the treatment of PJI's of the hip and knee caused by beta-lactam sensitive staphylococci treated with debridement, antibiotics, and implant retention (DAIR).

Methods: Ninety-one patients with beta-lactam sensitive PJI's of the hip and knee from two hospitals in Denmark received DAIR between January 1, 2004 - December 31, 2013. Fifty-one patients were postoperatively treated with beta-lactam antibiotic monotherapy (control group) at one institution, while at a second institution, 40 patients received combination therapy with a beta-lactam antibiotic and rifampicin. Patients were followed for two years from the date the DAIR procedure was carried out. Hazard Ratios (HR) were calculated using Cox proportional hazards regression analysis along with $95 \%$ confidence intervals $(\mathrm{Cl})$.

Results: No significant difference in arthroplasty removal within two years was found between the two groups; rifampicin group 13 (32.5\%) vs. control group 20 (39.2\%; $p=0.51)$. Comparing arthroplasty survival between the rifampicin group and the control group, a statistically significant difference was not found $((\mathrm{HR}) 0.81,95 \% \mathrm{Cl} 0.40-1.63, \mathrm{p}=0.55)$.

Conclusion: This study suggests that monotherapy with a beta-lactam is not inferior to combination therapy with a beta-lactam and rifampicin in treating staphylococcal PJI's with DAIR. We suggest that monotherapy with a beta-lactam in DAIR may be a safe option when treating staphylococcal PJI's.

\section{Introduction}

The treatment of acute periprosthetic joint infections (PJI) with surgical debridement, antibiotics, and retention of the implant (DAIR) is viable and safe(1-3). Since the start of the millennium, the number of PJls treated with combination therapy, including rifampicin, has increased with the aim to conserve the arthroplasty. The rationale for this strategy is evidence of adequate in vitro penetration of rifampicin in staphylococcal biofilm (4-6). A single mutation in Staphylococcus aureus may cause resistance towards rifampicin (7), hence the need to combine rifampicin with an additional antibiotic to prevent resistance development.

Only a few clinical studies exist comparing the treatment effect on PJI's with rifampicin in addition and absence with another antibiotic. A review from 2010 (8) found one randomized controlled trial (RCT) and eight retrospective studies. Likewise, a letter from Eisen et al. (9) has emphasized the poor level of evidence of combination therapy with rifampicin for the treatment of PJI.

The RCT (10) and five of the retrospective studies (11-15) compares the effect of combining rifampicin with a fluoroquinolone. 
This study aimed to retrospectively examine if monotherapy with beta-lactam antibiotic is inferior to combination therapy of a beta-lactam and rifampicin in the treatment of PJI's of the hip and knee caused by beta-lactam sensitive Staphylococcus species treated with DAIR.

\section{Material And Methods}

Patients were included from two orthopedic departments in the Capital Region of Denmark. One department used monotherapy with a beta-lactam as standard therapy of PJl's caused by staphylococci. In contrast, another orthopaedic department in the same region used a combination of a beta-lactam and rifampicin as standard therapy.

Patients were identified from diagnosis codes (ICD-10) and positive cultures with Staphylococcus aureus and coagulase-negative staphylococci (CoNS) in the database from the respective departments of clinical microbiology. Infections caused by methicillin-resistant Staphylococcus aureus (MRSA) are uncommon in Denmark. Information about orthopaedic hospitalization, including antibiotic choice and dates of surgery, was extracted from the electronic patient records. Musculoskeletal Infection Society (MSIS) criteria for prosthetic joint infection were not in included, because of insufficient reporting.

Patients included in the study received surgery with debridement and retention between January 1, 2004 - December 31, 2013. Biopsies were taken preoperatively via the Kamme \& Lindeberg method(16). All patients needed a minimum of 1 out of 5 positive biopsies of Methicillin susceptible Staphylococcus aureus (MSSA), or two out of five positive biopsies of CoNS to be included in the study. All patients in the study were administered a beta-lactam antibiotic as monotherapy or in combination with rifampicin. Patients were followed two years from the first DAIR procedure. Only patients with primary total hip arthroplasties (THA) and total knee arthroplasties (TKA) were included. Thus, revision hip and knee arthroplasty patients were excluded as well as patients with osteosynthesis materials (cannulated screws, dynamic hip screw or intramedullary nail), hemiarthroplasty, or girdle stone status.

Patients were excluded if any other bacteria than $S$. aureus and CoNS were found in any of the biopsies at any DAIR surgery, including during implant removal. If patients were administered any other antibiotic than a beta-lactam or rifampicin after DAIR, the patients were excluded. Moreover, if patients were administered lifelong antibiotics to lower the bacterial load in the hope of retention of implant, they were excluded as well.

Standard protocol for DAIR in Denmark, is one stage debridement surgery with no use of local antibiotics and exchange of moveable parts such as the polyethylene liner. Intravenous antibiotics is administered for two weeks and subsequentially changed to peroral antibiotic which is given for three months. Both institutions follow this protocol.

Description of the cohort population characteristics included medians, Interquartile ranges (IQR), and frequency tables. Event-free survival was calculated from the first biopsies until arthroplasty removal, death, or the last day of follow-up, whichever came first. Hazard Ratios (HR) were calculated using Cox 
proportional hazards regression analysis along with 95\% confidence intervals (Cl) for arthroplasty removal with follow-up periods of two years. The HR was adjusted for age, sex, location of prosthetic joint, diabetes, cardiovascular disease, and chronic obstructive pulmonary disease.

\section{Results}

A total of 342 patients with diagnosis codes (ICD-10-CM: DM00, DM01, DT84, DS71) corresponding to infections in the knee or hip were primarily identified for further investigation; 197 patients were from the hospital which treated PJI's primarily with combination therapy, while 145 patients were from the hospital treated with beta-lactam monotherapy. In total, 234 patients were excluded after the initial assessment. Sixty-Eighty patients were excluded because of removal of implant during the first surgical debridement. Fifty-six patients were excluded due to the use of antibiotics that were not a beta-lactam or rifampicin or had the presence of polymicrobial growth in the bacterial cultures.

Forty-two had either osteosynthesis material instead of an arthroplasty or not have an arthroplasty whatsoever and were therefore excluded. Thirty-nine patients had insufficient records or were duplicates.

Twelve patients were excluded due to insufficient DAIR regime such as not receiving surgery or administered antibiotics. Five patients were administered antibiotics intended for lifetime usage were excluded as well.

Consequently, 251 patients were excluded in total, and a total of ninety-one patients was included in the study, with forty patients receiving combination therapy with rifampicin vs. fifty-one patients in the control group (receiving only a beta-lactam antibiotic).

The results are summarized in Table 1. 
Table 1

\begin{tabular}{|c|c|c|c|}
\hline & $\begin{array}{l}\text { Control group } \\
(n=51)\end{array}$ & $\begin{array}{l}\text { Rifampicin group } \\
(n=40)\end{array}$ & P-value \\
\hline Age, median (IQR) & $73(64-80)$ & $68.5(63.5-76.5)$ & \\
\hline Sex, females (\%) & $30(58.8)$ & $25(62.5)$ & 0.72 \\
\hline More than one DAIR surgery (\%) & $9(17.7)$ & $4(10.0)$ & 0.30 \\
\hline Location of arthroplasty & & & 0.73 \\
\hline - Knee (\%) & $17(33.3)$ & $12(30.0)$ & \\
\hline - Hip (\%) & $34(66.7)$ & $27(70.0)$ & \\
\hline \multicolumn{4}{|l|}{ Comorbidities } \\
\hline - Cardiovascular (\%) & $8(15.7)$ & $2(5)$ & 0.11 \\
\hline - Diabetes (\%) & $3(5.9)$ & $2(5.0)$ & 0.86 \\
\hline - COPD (\%) & $2(3.9)$ & $3(7.5)$ & 0.65 \\
\hline $\begin{array}{l}\text { Arthroplasty removal within } 2 \text { years } \\
(\%)\end{array}$ & 20 (39.2) & 13 (32.5) & 0.51 \\
\hline Onset of infection (17) & & & 0.02 \\
\hline - Early (<3 months) & $27(52.9)$ & $33(82.5)$ & \\
\hline - Delayed (3-12 months) & $11(21.6)$ & $2(5.0)$ & \\
\hline - Late (> 12 months) & $10(19.6)$ & $4(10.0)$ & \\
\hline - Missing primary OP date & $3(5.9)$ & $1(2.5)$ & \\
\hline Staphylococcus aureus & $45(88.2)$ & $38(95.0)$ & 0.45 \\
\hline
\end{tabular}

\begin{tabular}{|lllll|}
\hline \multicolumn{2}{|l}{ Crude } & & \multicolumn{2}{l|}{ Adjusted* } \\
\hline & HR $(95 \% \mathrm{Cl})$ & P-value & HR $(95 \% \mathrm{Cl})$ & P-value \\
\hline Non-rifampicin & Ref. & - & Ref. & - \\
\hline Rifampicin & $0.81(0.40$ to 1.63$)$ & 0.55 & $0.77(0.37-1.60)$ & 0.49 \\
\hline $\begin{array}{l}\text { *Adjusted for age, sex, location of arthroplasty, diabetes, cardiovascular disease, and chronic } \\
\text { obstructive pulmonary disease. }\end{array}$ & & & \\
\hline
\end{tabular}

The rifampicin groups' median in age was 68.5 (63.5-76.5) years, and in the control group, 73 (64-80) years. The proportion of women in the two groups was similar ( $62.5 \%$ versus $58.8 \%)$. Four patients (10\%) in the rifampicin group had more than one DAIR surgery, whereas nine patients (17.7\%) had more than 
one DAIR surgery in the control group. In the rifampicin group, 17 (33.3\%) PJIs were located to the knee, and 34 PJIs (66.7\%) were located to the hip. PJIs in the control group were located to the knee in $12(30 \%)$ patients and $27(70 \%)$ in the hip.

When comparing survival of the prosthesis in the two groups, no significant difference in arthoplasty removal within two years was found between the two groups; rifampicin group 13 (32.5\%) vs. control group 20 (39.2\%; $p=0.51)$.

Looking at the onset of PJI from the primary operation date, patients were categorized into early-onset (< 3 months, delayed onset (3-12 months) and late-onset (> 12 months)(17). Moreover, patients without the primary operation date were included in the comparison. Overall, patients in the rifampicin group had an earlier diagnosis of infection compared to the control group $(p=0.02)$.

S. aureus was more frequent in the biopsies compared to CoNS, and there was no significant difference in the prevalence of biopsies with S.aureus between the rifampicin patients, 38 (95.0\%), and the patients in the control group, $45(88.2 \%, p=0.45)$.

We found no significant difference in arthroplasty survival when comparing the two group, neither in the crude analysis (HR $0.81,95 \% \mathrm{Cl} 0.40-1.63, \mathrm{p}=0.55)$ or when adjusting for age, sex, location of prosthetic joint, diabetes, cardiovascular disease, and chronic obstructive pulmonary disease (HR $0.77,95 \% \mathrm{Cl} 0.37$ $1.60, p=0.49)$.

\section{Discussion}

According to the results, the patients in the rifampicin group and control group were similar concerning age, sex, and comorbidities. Moreover, the patients in the two groups were comparable in terms of the proportion of patients having more than one surgery, and that the main location of PJI's was the hip with the bacteria present in the infections being $S$. aureus. No statistical difference $(p=0.51)$ in the number of patients who had their prosthesis removed within two years was observed, nor was there any difference in $\mathrm{HR}(0.81,95 \% \mathrm{Cl} 0.40-1.63)$, even though we adjusted for comorbidities (adjusted $\mathrm{HR} 0.77,95 \% \mathrm{Cl}$ 0.37 - 1.60). Nine patients in the control group vs. four in the rifampicin group had more than one DAIR procedure which could be due to different hospital treatment strategies. In this study, DAIR was primarily carried out on hip arthroplasties (70\%), which could be due to surgeons being more prone to debridement and retention in the hip because of the larger soft tissue envelope around the hip. Interestingly, and probably due to different strategies at the two hospitals, the rifampicin group had a higher proportion of patients with early onset of infection compared to the control group (82.5\% vs. 52.9\%). Early-onset of infection has shown higher success rates of DAIR in PJI(1), and this observation would have favoured a better outcome in the rifampicin group, which we did not see.

Although this one of the largest studies to date looking into this issue, one explicit limitation of this study is the relatively small number of patients in each group. Although 342 patients with infection around hip and knee were primarily selected, only a limited amount ( $\mathrm{N}=91)$ was found eligible for enrolment. Thus, 
one could argue that the small sample size is not considered representative of the population. Hence the results may not be generalized to a larger population. Due to the retrospective design of our study, the results are prone to selection bias and inaccuracies in reporting.

Arthroplasty revision surgery in Denmark is usually performed by a highly trained orthopaedic specialist with a standardized methodology when performing the surgery. The methodology of the debridement and retention could have and impact of outcome if there is difference in the amount of saline solution used or if hydrogen peroxide is utilized during debridement(18). However, it is assumable that these are minor differences that most likely do not confound the results of this study. Additionally, we did not record perioperatively surgical risk factors such as if pus was found in the surgical field, and the duration of surgery. One aspect which may be of significance in infection risk and management is the number of surgeries carried out at each hospital; a higher number raises the risk of infection, though it may increase the quality of surgery and management of PJI's. In our study, the control group had a higher amount.

Another critical factor is the perioperative exchange of polyethylene liner or lack of. Unfortunately, this was not stated in most the patients' records, and therefore not considered in the analyses.

A strength of the study is the adjustment for comorbidities. During the patient records assessment, only an insufficient number of patients who were otherwise immunocompromised was found. Thus, patients were not adjusted for this. Other patient-related factors that were not feasible to obtain that could potentially have had an impact is high Body Mass Index (BMI), high American Society of Anaesthesiologists (ASA) score, smoking status, and alcohol consumption.

Other strengths in this study, despite the relatively small sample size, has by one of the biggest sample size vis-à-vis research on this issue. This constitutes yet another strength. The clarity of the study, i.e., only patients included in the study, had S. aureus and CoNS PJI's in THA's and TKA's, and the utilization of only beta-lactam antibiotics and rifampicin in the postoperative regime in the treatment of PJI after DAIR. In contrast, Zimmerli's study(10) contained a more broad spectrum of infections, including infections in osteosynthesis material, osteomyelitis, and PJI's. Moreover, the patients at the two hospitals in our study seem comparable in most aspects, such as socioeconomic status.

In the RCT by Zimmerli et al., the effect of ciprofloxacin on staphylococcal PJI's with and without the addition of rifampicin was examined (10). The patients were followed for two years. All the patients went through DAIR with the antibiotics administered postoperatively. There were 12 patients in each group. All patients receiving rifampicin were cured $(100 \%)$, while only seven patients $(58 \%)$ who were treated with ciprofloxacin monotherapy were cured. Notably, ciprofloxacin is a poor agent towards $S$. aureus; hence four out of the five staphylococcus isolates with failed treatment had developed resistance towards ciprofloxacin, and consequently, the patients in the control group did not receive adequate antibiotic treatment.

The use of penicillinase-resistant beta-lactam antibiotics as monotherapy is a well-known and effective treatment towards infections caused by sensitive $S$. aureus. Westberg et al. treated 10 out of 10 early 
staphylococcal PJI's successfully with DAIR and beta-lactam antibiotics (19). Thus another clear difference and strength of our study compared to Zimmerli's (10) study is the use of a beta-lactam compared to a quinolone in the treatment of S.aureus PJI's.

In a review from Clinical Microbiology Reviews concerning Staphylococcus aureus infections (20), the same randomized study by Zimmerli et al. was found (10); further studies were not found. In this review, the authors express the same concern and critique, as we have mentioned above.

Norden et al compared the effect of a penicillinase-resistant beta-lactam antibiotic (nafcillin) as a monotherapy and in combination with rifampicin (21). This study showed no difference in outcome when treated with monotherapy or in combination with rifampicin. The study included 12 patients with chronic osteomyelitis who had a surgical debridement and, additionally, removal of the implant if present. The patients were followed for six months after the treatment ended. Thus, the patients did not retain an implant while receiving antibiotic treatment.

In a recently conducted multicentre RCT from Norway, 48 patients with PJI's with Staphylococcus aureus or CoNS were treated with DAIR, and either monotherapy cloxacillin or vancomycin treatment, or in combination with rifampicin. The study concluded that adding rifampicin did not affect the cure rate of a DAIR procedure in patients with acute staphylococcal infection (22), thus supporting the findings in our study.

In vitro animal studies that compare the effect of combination therapy with beta-lactam antibiotics have revealed conflicting results(8). Thus, a combination of beta-lactam and rifampicin in the treatment of PJI's after DAIR is only supported by weak evidence. Furthermore, rifampicin is costly and is known to have potential adverse effects such as hepatotoxicity and drug interactions(23). We suggest that monotherapy with a beta-lactam in DAIR is a safe option when treating staphylococcal PJI's in TKA's and THA's.

\section{Conclusion}

In conclusion, this study suggests that monotherapy with a beta-lactam is not inferior to combination therapy of beta-lactam and rifampicin in the treatment of PJI's with DAIR caused by Staphylococcus aureus or CoNS. Additional studies are warranted to confirm our results, ideally a large randomized controlled clinical trial.

\section{Abbrevations}

PJI: Periprosthetic joint infections; DAIR: Debridement, antibiotics, and retention of the implant; RCT: Randomized controlled trial; CoNS: Coagulase-negative staphylococci; MRSA: methicillin-resistant Staphylococcus aureus; THA: Total hip arthroplasty; TKA: Total knee arthroplasty; HR: Hazard Ratios; Cl: confidence interval. 


\section{Abbreviations}

PJI: Periprosthetic joint infections; DAIR: Debridement, antibiotics, and retention of the implant; RCT: Randomized controlled trial; CoNS: Coagulase-negative staphylococci; MRSA: methicillin-resistant Staphylococcus aureus; THA: Total hip arthroplasty; TKA: Total knee arthroplasty; HR: Hazard Ratios; Cl: confidence interval.

\section{Declarations}

Acknowledgements

Not applicable

Authors' contributions

All the authors have made a significant development of the study and finishing the manuscript. The authors read and approved the final manuscript.

Funding

No funding was required

Availability of data and materials

The datasets used in the study are available from the corresponding author on reasonable request.

Ethics approval and consent to participate

Not applicable

Consent for publication

Not applicable

Competing interests

The authors have no conflict of interest.

\section{References}

1. Ottesen CS, Troelsen A, Sandholdt H, Jacobsen S, Husted H, Gromov K. Acceptable Success Rate in Patients With Periprosthetic Knee Joint Infection Treated With Debridement, Antibiotics, and Implant Retention. J Arthroplasty. 2019. 
2. Mont MA, Waldman B, Banerjee C, Pacheco IH, Hungerford DS. Multiple irrigation, debridement, and retention of components in infected total knee arthroplasty. J Arthroplasty. 1997.

3. Geurts JAP, Janssen DMC, Kessels AGH, Walenkamp GHIM. Good results in postoperative and hematogenous deep infections of 89 stable total hip and knee replacements with retention of prosthesis and local antibiotics. Acta Orthop. 2013.

4. Dunne WM, Mason EO, Kaplan SL. Diffusion of rifampin and vancomycin through a Staphylococcus epidermidis biofilm. Antimicrob Agents Chemother. 1993.

5. Zheng Z, Stewart PS. Penetration of rifampin through Staphylococcus epidermidis biofilms. Antimicrob Agents Chemother. 2002.

6. Singh R, Ray P, Das A, Sharma M. Penetration of antibiotics through Staphylococcus aureus and Staphylococcus epidermidis biofilms. J Antimicrob Chemother. 2010.

7. Bals MG, Filipescǔ S. The antistaphylococcal activity of Rifampicin. Arzneimittel-Forschung/Drug Res. 1969.

8. Forrest GN, Tamura K. Rifampin combination therapy for nonmycobacterial infections. Clinical Microbiology Reviews. 2010.

9. Eisen DP, Denholm JS. Recommendations for rifampicin therapy of staphylococcal infection in infectious diseases society of America prosthetic joint infection guidelines are not supported by available literature. Clinical Infectious Diseases. 2013.

10. Zimmerli W, Widmer AF, Blatter M, Frei R, Ochsner PE. Role of rifampin for treatment of orthopedic implant-related staphylococcal infections: A randomized controlled trial. J Am Med Assoc. 1998.

11. Barberán J, Aguilar L, Giménez MJ, Carroquino G, Granizo JJ, Prieto J. Levofloxacin plus rifampicin conservative treatment of 25 early staphylococcal infections of osteosynthetic devices for rigid internal fixation. Int J Antimicrob Agents. 2008.

12. Laffer RR, Graber P, Ochsner PE, Zimmerli W. Outcome of prosthetic knee-associated infection: Evaluation of 40 consecutive episodes at a single centre. Clin Microbiol Infect. 2006.

13. Choong PFM, Dowsey MM, Carr D, Daffy J, Stanley P. Risk factors associated with acute hip prosthetic joint infections and outcome of treatment with a rifampinbased regimen. Acta Orthop. 2007.

14. Aboltins CA, Page MA, Buising KL, Jenney AWJ, Daffy JR, Choong PFM, et al. Treatment of staphylococcal prosthetic joint infections with debridement, prosthesis retention and oral rifampicin and fusidic acid. Clin Microbiol Infect. 2007.

15. Donaldson AD, Chan RC, Gosbell IB. Community-acquired methicillin-resistan Staphylococcus aureus in bone and joint infections: Development of rifampicin resistance [2]. Medical Journal of Australia. 2006.

16. Kamme C, Lindberg L. Aerobic and anaerobic bacteria in deep infections after total hip arthroplasty: differential diagnosis between infectious and non-infectious loosening. Clin Orthop Relat Res. 1980.

17. Tande AJ, Patel R. Prosthetic joint infection. Clin Microbiol Rev. 2014. 
18. Lu M, Hansen EN. Hydrogen Peroxide Wound Irrigation in Orthopaedic Surgery. J Bone Jt Infect. 2016.

19. G MW,B. F. S. Early prosthetic joint infections treated with debridement and implant retention. Acta Orthopaedica. 2012.

20. Tong SYC, Davis JS, Eichenberger E, Holland TL, Fowler VG. Staphylococcus aureus infections: Epidemiology, pathophysiology, clinical manifestations, and management. Clin Microbiol Rev. 2015.

21. Norden CW, Fierer J, Bryant RE, Palmer DL, Jones SM, Bird CB, et al. Chronic staphylococcal osteomyelitis: Treatment with regimens containing rifampin. Rev Infect Dis. 1983.

22. Karlsen ØE, Borgen P, Bragnes B, Figved W, Grøgaard B, Rydinge J, et al. PJI RCT rifampicina 2020. 2020;2:1-9.

23. Riedel DJ, Weekes E, Forrest GN. Addition of rifampin to standard therapy for treatment of native valve infective endocarditis caused by Staphylococcus aureus. Antimicrob Agents Chemother. 2008;52(7):2463-7. 